\title{
An Observational Study Regarding Usage of Favipiravir for COVID-19 Therapy
}

\author{
Md Azizul Islam ${ }^{1}$, AKM Faizul Huq ${ }^{2}$, Azizur Rahman², Md Sakirul \\ Islam Khan ${ }^{3}$, Mamun Al Mahtab ${ }^{4}$ and Sheikh Mohammad Fazle \\ Akbar $^{5 *}$ \\ ${ }^{1}$ Directorate General of Medical Services, Bangladesh Armed Forces, Dhaka, \\ Bangladesh \\ ${ }^{2}$ Department of Medicine, Combined Military Hospital, Dhaka, Bangladesh \\ ${ }^{3}$ Department of Anatomy and Embryology, Ehime University Graduate School of \\ Medicine, Ehime, Japan \\ ${ }^{4}$ Department of Hepatology, Bangabandhu Sheikh Mujib Medical University, Dhaka, \\ Bangladesh \\ ${ }^{5}$ Department of Gastroenterology and Metabology, Ehime University Graduate \\ School of Medicine, Ehime, Japan \\ *Corresponding Author: Sheikh Mohammad Fazle Akbar, Department of \\ Gastroenterology and Metabology, Ehime University Graduate School of Medicine, \\ Ehime, Japan.
}

\author{
Received: October 03, 2020 \\ Published: October 22, 2020 \\ (C) All rights are reserved by Sheikh \\ Mohammad Fazle Akbar., et al.
}

\footnotetext{
Abstract

Background: A pandemic of severe acute respiratory syndrome coronavirus 2 (SARS-CoV-2) causing coronavirus disease 2019 (COVID-2019) has shattered the globe with no prophylactic vaccine or anti-SARS-CoV-2 medication inducing a morbidity of more than 75 million patients and mortality of more than one million individuals in the world.

Aims: This open level observational study has been designed to assess the therapeutic potentiality of favipiravir, an antiviral drug, originally developed for another coronavirus in patients with mild COVID-19.

Materials and Methods: A total of 45 COVID-19 patients positive for (SARS-CoV-2) confirmed by polymerase chain reaction (PCR) of nasal swab were enrolled in this study in Combined Military Hospital (CMH), Dhaka, Bangladesh. The patients were provided with standard of care (SoC) plus favipiravir for 10 days.

Results: No notable adverse event was recorded due to usage of favipiravir in these patients during the observation period. All patients became negative for SARS-CoV-2 and they were discharged from the hospital within a mean period of 12.4 days (the shortest period being 3 days and longest was 30 days).

Conclusion: Although this is an open level study, it seems that favipiravir is safe and effective for treatment of mild COVID-patients. However, a double-blind, controlled study with large sample size would be required to validate its clinical usage and registration. Keywords: Severe Acute Respiratory Syndrome 2 (SARS-CoV-2); Coronavirus Disease 2019 (COVID-19); Favipiravir
}

\section{Introduction}

Severe acute respiratory syndrome 2 (SARS-CoV-2) is the newly evolving human coronavirus infection that causes coronavirus disease 2019 (COVID-19) [1]. The infection was first reported in China in December 2019 and spread rapidly to all over the world; more than 7.5 million confirmed infections and more than one mil- 
lion deaths around the world in last 10 months [2,3]. There is no prophylactic vaccine for this virus. Although at least seven months have passed after initial reporting of the virus, and it seems that a vaccine of some potentiality may be available within next $6-12$ months. In this pretext, various approaches have been taken for containment the spread of this virus around the world such as 'Testing, Contact Tracing and Treatment (3Ts)' as well as 'Contact, Crowd and Congestion (3Cs)' concepts. When all realities are compiled together, it seems that pandemic of COVID-19 is a challenge to the international community that may not be addressed with conventional approaches $[4,5]$.

The clinical course of the COVID-19 patients is highly variable and difficult to predict. A major proportion of COVID-19 patients remain completely asymptomatic and the infection of these persons is detected as a chance finding when SARS-CoV-2 is searched in these patients as contacts of other COVID-19 patients [6,7]. The next, another group of SARS-CoV-2-infected patients exhibit with mild or moderate symptoms and majority of these patients may not need any specific therapy and these patients recover spontaneously. Although the molecular facts indicate that the pathogenesis of COVID-19 is yet to be explored, asymptomatic COVID-19 patients as well as COVID-19 patients with mild and moderate symptoms may suddenly develop serious illness with intractable complications like acute respiratory distress syndrome (ARDS) and pneumonias as well as various other multiorgan pathologies [8-11].

When all these facts are properly analyzed, it become apparent that there is pressing need for development of drugs for containment of SARS-CoV-2 and control of complications of COVID-2019. Scientific oversights indicate that development of a new drug for COVID-19 is a time-consuming factor and as a practical approach different available antiviral drugs are now being tested for COVID-19 patients [12-16].

More than 300 clinical trials are ongoing with various exiting antiviral and immunomodulating agents in various stages of treatment of COVID-19. The most frequently used agents include chloroquine, hydroxychloroquine, lopinavir/ritonavir, favipiravir and remdesivir, nitazoxanide and ivermectin [17-21]. We have reported two observational studies in which safety and efficacy of hydroxychloroquine with two antibiotic combinations were assessed in COVID-19 patients in Bangladesh [22,23]. As next step, in the present communication, we have assessed the safety and efficacy of favipiravir for COVID-19 patients in Bangladesh. These observational studies would bring us close to developing proper effective treatment regimen for COVID-19 patients in Bangladesh.

\section{Patients and Methods}

\section{Patients}

This observational study was conducted under the supervision of Combined Military Hospital (CMH), Dhaka, Bangladesh. The study is a single center and prospective in nature. The study design has been approved by the hospital authority and informed consent was taken from all patients. The inclusion criteria included positive for SARS-CoV-2 and adult having an age over 18 years. The exclusion criteria included negativity for SARS-CoV-2, age below 18 years, and harboring severe forms of pneumonia. All patients in this cohort had mild form of COVID-19. SARS-CoV-2 positivity was evaluated by polymerase chain reaction (PCR) of nasal swab. The criteria for discharge from the hospital included to be negative for SARS-CoV-2 in two consecutive assessments, 24 hours apart and being free of symptoms and signs of COVID-19, if any. A total of 45 patients could be finally analyzed on the basis of inclusion and exclusion criteria.

\section{Therapeutic regimen}

All patients received standard of Care (SoC) therapy as per recommendation of the attending physician based on their clinical conditions and pathological evaluations. SoC usually included drugs of symptomatic maneuvers. Antipyretic drugs were given for fever and pain killers for reducing pain. In some cases, antibiotic was given when infection was considered. Oxygen was given when oxygen concentration in the blood persistently reduced below $94 \%$ reduced and patients had respiratory difficulty. In addition to the SoC, all patients received one antiviral drug, favipiravir. It was given at dose of $1600 \mathrm{mg}$, twice daily, on day 1 and this was followed by $600 \mathrm{mg}$, twice daily, from $2^{\text {nd }}$ to $10^{\text {th }}$ days. If a patient recovered from symptoms of COVID-19 and became negative for SARS-CoV-2, the patient was subjected to discharge. All patients maintained a constant contact with the $\mathrm{CMH}$ so that any future adverse events of the virus or re-infection could be addressed.

\section{Trial procedure}

$\mathrm{CMH}$ is a highly organized hospital and all patients were properly assessed about vital signs, and physical examination during admission. Also, all new symptoms and aggravation of pre-existing symptoms were monitored. Peripheral blood was taken from each patient to check complete blood picture, levels of hemoglobulin, 
creatinine, bilirubin, and alanine aminotransferase (ALT) to develop insight about the condition of vital organs. X-ray of chest was accomplished as and when necessary. Oxygen saturation was monitored regularly and all measures were adopted to provide oxygen supply. Also, ventilator facilities were at hand for use if indicated.

\section{Statistical analysis}

The data have been shown as mean and standard deviation as well as median and range. For the statistical analysis, paired t test was used for normally distributed data. When the distribution was skewed, the Wilcoxon signed-rank test was used. A p value of less than 0.05 was regarded as statistically significant.

\section{Result}

After carefully considering the inclusion and exclusion criteria and on the basis of aim of objective of the study, a total of 45 patients were finally enrolled in this study. The majority of the patients were male $(\mathrm{N}=36)$. The age of the patients varied considerably and it ranged from 20 - 86 years. The mean age was 47.6 years. The patient's profile has been shown in table 1 . Twenty-five patients were below 50 years of age, whereas the rest 20 patients were comparatively older. The comorbidities of the patients have been shown in table 1. Diabetes mellitus was the most common comorbidity and this was detected in 9 patients. Hypertension and hypothyroidism were seen in 2 and 1 patients, respectively. Table 1 profiles treatment approaches and clinical outcomes of patients with COVID-19.

\begin{tabular}{|l|c|}
\hline Variables & Total patients \\
\hline Number of patients & $\mathbf{4 5}$ \\
\hline Characteristics of patients & \\
\hline Age, years & \\
\hline Age group & $25(56)$ \\
\hline 20 - 50 & $20(44)$ \\
\hline$\geq 51$ & \\
\hline Sex & $9(20)$ \\
\hline Female & $36(80)$ \\
\hline Male & \\
\hline $\begin{array}{l}\text { Comorbidities and preexisting } \\
\text { conditions }\end{array}$ & $1(2)$ \\
\hline Hypertension & $9(20)$ \\
\hline Diabetes Mellitus & $2(4)$ \\
\hline Hypothyroidism & \\
\hline Presenting symptoms & $45(100)$ \\
\hline Cough & \\
\hline
\end{tabular}

\begin{tabular}{|l|c|}
\hline Headache & $10(22)$ \\
\hline Myalgia & $8(18)$ \\
\hline Loose motion & $4(9)$ \\
\hline Anosmia & $2(4)$ \\
\hline Body temperature- ${ }^{\circ} \mathrm{F}$ & $98.4 \pm 1.5(98 ; 96-101)$ \\
\hline$\geq 99.5$ & $4(9)$ \\
\hline Heart rate - beats/min & $81 \pm 10(78 ; 68-10)$ \\
\hline Respiratory rate - breaths/min & $13 \pm 1(13 ; 10-14)$ \\
\hline Oxygen saturation - \% & $96 \pm 1(96 ; 94-98)$ \\
\hline$\leq 94$ & $1(2)$ \\
\hline Treatment approaches & $4(9)$ \\
\hline Antipyretic drug & $5(100)$ \\
\hline Antibiotic (azithromycin) & $45(100)$ \\
\hline Favipiravir & $12.4 \pm 5.9(13 ; 3-30)$ \\
\hline Clinical outcomes & $45(100)$ \\
\hline Duration of hospital stay-day & \\
\hline Discharged & \\
\hline
\end{tabular}

Table 1: Profiles treatment approaches and clinical outcomes of patients with COVID-19.

Data of age, body temperature, heart rate, respiratory rate, and duration of hospital stay are shown as mean \pm standard deviation (median and range). Other values are number and parenthesis indicates $\%$ of the group.

The presenting symptoms were also highly variable and have been shown in table 1 . Cough was a common complain of all patients. Others reported symptoms were compatible with common cold or COVID-19, such as headache, myalgia, loose motion, anosmia. The oxygen saturation was mostly within normal range except in one patient with $94 \%$ oxygen saturation. Different parameters of blood for assessing general conditions, liver function test, kidney functions and electrolyte levels have been cited in table 2. Data of different parameters have been at two time points, one during admission and the other 7 days after taking favipiravir. Depending on the condition of the patient, they were given oxygen supplementation. Steroid was given based on the decision of the attending physicians. The patients did not receive convalescent plasma or immunomodulating agent.

The study was designed to assess two important points about favipiravir: (1) the safety of the drug and (2) the antiviral potentiality of the drug in COVID-19 patients. To accomplish this goal, we checked different parameters of safety in different time points. 
The levels of liver functions [alanine aminotransferase (ALT) and aspartate aminotransferase (AST), serum bilirubin], kidney functions [serum creatinine], and those of different electrolytes have been plotted at two points, just before admission and one week after admission. The levels of ALT were higher in 17 patients during admission. In 10 patients, the levels of ALT were elevated during therapy. In 7 patients, the levels of ALT reduced during therapy and 1 of them reverted to within normal range. The levels of other serological markers regarding kidney functions or serum electrolyte remain unaltered after receiving favipiravir.
The second objective was about antiviral potentiality of favipiravir. All patients became negative for SARS-CoV-2 by PCR during discharge. The average during of discharge after admission was 23.3 days and the range was 3-30 days. Although early discharge was done for some patients, all patients were instructed to take favipiravir for proper duration. As this is an observational study, there is no comparable group in this cohort. Thus, there may some patients who might be negative for SARS-CoV-2 as a part of natural course even without any treatment.

There has been no death in this cohort and none required any intensive care unit facility.

\begin{tabular}{|l|c|c|}
\hline Variables & Day of admission & 7 Days after Admission \\
\hline Pathological features & & \\
\hline Hemoglobin concentration $(\%)$ & $12.9 \pm 2.4(12.4 ; 9.0-19.4)$ & $12.8 \pm 1.4(12.8 ; 10.0-15.3)$ \\
\hline WBC $\left(\mathrm{mm}^{3}\right)$ & $7369 \pm 3685(6300 ; 2400-15000)$ & $9490 \pm 4478(8500 ; 3000-19000)$ \\
\hline Neutrophil (\%) & $66 \pm 17(68 ; 40-90)$ & $76 \pm 13 *(81 ; 48-95)$ \\
\hline Platelet (mm $\left.{ }^{3}\right)$ & $224 \pm 139(160 ; 91-666)$ & $287 \pm 131(247 ; 141-631)$ \\
\hline Bilirubin (mg/dL) & $0.69 \pm 0.56(0.50 ; 0.30-3.20)$ & $0.77 \pm 0.42(0.73 ; 0.30-1.60)$ \\
\hline ALT (U/L) & $81 \pm 85(58 ; 15-355)$ & $81 \pm 66(45 ; 11-300)$ \\
\hline AST (U/L) & $54 \pm 23(61 ; 19-107)$ & $41 \pm 20(37 ; 21-121)$ \\
\hline Albumin (g/L) & $3.8 \pm 0.6(3.8 ; 2.8-5.0)$ & $3.6 \pm 0.4(3.6 ; 2.9-4.4)$ \\
\hline Creatinine (mg/dL) & $1.3 \pm 2.3(0.8 ; 0.6-12)$ & $1.1 \pm 2.1(0.7 ; 0.6-12)$ \\
\hline Sodium (mEq/L) & $136 \pm 5(137 ; 122-143)$ & $138 \pm 5(139 ; 128-150)$ \\
\hline Potassium (mEq/L) & $4.3 \pm 0.6(4.2 ; 3.3-5.8)$ & $4.2 \pm 0.8(4.2 ; 1.0-5.5)$ \\
\hline Chloride (mEq/L) & $95.8 \pm 5.1(97 ; 81-109)$ & $96.8 \pm 3.4(97 ; 90-105)$ \\
\hline
\end{tabular}

Table 2: Pathological features of patients with COVID-19.

Data are shown as mean \pm standard deviation (median and range). WBC: White Blood Cell; S. ALT: Alanine Transaminase; AST: Aspartate Transaminase. Statistical significance was considered when p value was $<0.05$ and shown as * mark.

\section{Discussion}

Outbreak and pandemic of coronavirus SARS-CoV-2 in 2019/2020 have challenged the present and future of global health and health care delivery system of almost all countries of the world $[2,3]$. In fact, with some minor exceptions, most of the countries of the world, both developed and developing, both rich and poor countries have measurably failed to take strong strategies for containment of this pandemic [24]. Because an effective vaccine against the virus would not be available soon, pharmacological strategy is required to combat the virus. This has induced a global competition for developing antiviral drugs for COVID-19.
There are a number of candidate drugs that may inhibit replication of SARS-CoV-2, such as inhibitors of TMPRSS2 serine protease, inhibitors of angiotensin-converting enzyme 2 (ACE2), chloroquine and hydroxychloroquine, and off-label antiviral drugs, such as the nucleotide analogue remdesivir, HIV protease inhibitors lopinavir and ritonavir, broad-spectrum antiviral drugs arbidol and favipiravir as well as antiviral phytochemicals [19-21]. However, Despite the worsening trends of COVID-19, no drugs are validated to have significant efficacy in clinical treatment of COVID-19 patients in large-scale studies. 
In this study, we used favipiravir in a cohort of mostly mild COVID-19 patients at a highly organized hospital of Bangladesh. Favipiravir is an RNA-dependent RNA-polymerase inhibitor and has been licensed for common cold and flu like diseases in Japan. Recently, after COVID-19 epidemic, the drug has been approved for usage in China [25]. Japan has initiated several clinical trials with favipiravir in many countries as well as in Japan. The data of these clinical trials would pave the way for its approval by Japanese Pharmaceutical and Medical Device Agency (equivalent as Japanese Federal Drug Administration [FDA]) and United States Federal Drug Administration (USFDA). The Government of Bangladesh has permitted the use of favipiravir for COVID-19 patients. The study presented here have shown that the drug is safe in patients with COVID-19 with no apparent adverse effects. The next, outcome also revealed that the drug can ensue negativity of SARS-CoV-2 in COVID patients. The data about favipiravir in COVID-19 patients are encouraging and this support our previous studies in which hydroxychloroquine showed potent antiviral effect in mild and moderate patients of COVID-19 [22,23]. This is an extremely important issue as if COVID-19 patients with mild symptoms may be reverted to SARS-CoV-2 negativity, severe and complicated patients of COVID-19 would be highly limited.

\section{Conclusion}

However, there are some notable limitations of the present study. The first, the sample size is small. The next, there has been no control group in this study. In addition, long term follow up data is not available at this point. However, the patients remain in follow up and we would compile the data about adverse effects in future. Also, concerns remain about usage of favipiravir in children and pregnant mothers. Another major concern prevails about hepatotoxic effects of favipiravir. In this cohort, some patients already had elevated ALT, however, favipiravir did not induced fulminant hepatitis or liver failure in these patients. Follow up of these patients would unveil new information about hepatotoxic effect of favipiravir.

Taken all pons and cons of this observational study, it appears that there should be a large-scale control trial to assess the safety and real efficacy of favipiravir for treatment of COVID-19 patients and in fact, several study of these matures are running in different countries including Japan. We have added some important inputs in this regard.

\section{Bibliography}

1. Zhu N., et al. "A novel coronavirus from patients with pneumonia in China, 2019". The New England Journal of Medicine 382 (2020): 727-733.

2. Zhou P., et al. "A pneumonia outbreak associated with a new coronavirus of probable bat origin". Nature 579 (2020): 270273.

3. WHO Coronavirus disease (COVID-2019) situation reports (2020).

4. Perez GIP and Abadi ATB. "Ongoing Challenges Faced in the global control of COVID-19 pandemic". Archives of Medical Research (2020).

5. Brock RL and Laifer LM. "Family Science in the Context of the COVID-19 Pandemic: solutions and new directions". Fam Process 59.3 (2020): 1007-1017.

6. Gao Z., et al. "A systematic review of asymptomatic infections with COVID-19". Journal of Microbiology, Immunology and Infection (2020).

7. Rivett L., et al. "Screening of healthcare workers for SARSCoV-2 highlights the role of asymptomatic carriage in COVID-19 transmission". Elife 9 (2020): e58728.

8. Kim GU., et al. "Clinical characteristics of asymptomatic and symptomatic patients with mild COVID-19". Clinical Microbiology and Infection 26.7 (2020): 948.e1-948.e3.

9. Castagnoli R., et al. "Severe Acute Respiratory Syndrome Coronavirus 2 (SARS-CoV-2) Infection in Children and Adolescents: A Systematic Review". JAMA Pediatrics (2020).

10. Henry BM., et al. "Hematologic, biochemical and immune biomarker abnormalities associated with severe illness and mortality in coronavirus disease 2019 (COVID-19): a meta-analysis". Clinical Chemistry and Laboratory Medicine 58.7 (2020): 1021-1028.

11. Alhazzani W., et al. "Surviving Sepsis Campaign: guidelines on the management of critically ill adults with Coronavirus Disease 2019 (COVID-19)". Intensive Care Medicine 46.5 (2020): 854-887.

12. Xie P., et al. "Severe COVID-19: A Review of Recent Progress With a Look Toward the Future". Front Public Health 8 (2020): 189. 
13. Japan ECMOnet for COVID-19. "Japan ECMOnet for COVID-19: telephone consultations for cases with severe respiratory failure caused by COVID-19. Version 2". Journal of Intensive Care 8 (2020): 24.

14. Sun P., et al. "Understanding of COVID-19 based on current evidence". Journal of Medical Virology 92.6 (2020): 548-551.

15. Mehta N., et al. "Pharmacotherapy in COVID-19; A narrative review for emergency providers". American Journal of Emergency Medicine 38.7 (2020): 1488-1493.

16. Tu YF., et al. "A Review of SARS-CoV-2 and the Ongoing Clinical Trials". International Journal of Molecular Sciences 21.7 (2020): 2657.

17. Jean SS., et al. "Treatment options for COVID-19: The reality and challenges". Journal of Microbiology, Immunology and Infection 53.3 (2020): 436-443.

18. Zhang W., et al. "The use of anti-inflammatory drugs in the treatment of people with severe coronavirus disease 2019 (COVID-19): The Perspectives of clinical immunologists from China". Journal of Clinical Immunology 214 (2020): 108393.

19. Gao J., et al. "Breakthrough: Chloroquine phosphate has shown apparent efficacy in treatment of COVID-19 associated pneumonia in clinical studies". Bio Science Trends 14.1 (2020): 7273.

20. Potì F., et al. "Treatments for COVID-19: emerging drugs against the coronavirus". Acta Bio Medica 91.2 (2020): 118-136.

21. Abd El-Aziz TM and Stockand JD. "Recent progress and challenges in drug development against COVID-19 coronavirus (SARS-CoV-2) - an update on the status". Infection, Genetics and Evolution 83 (2020): 104327.

22. Bhuyan MAR., et al. "Treatment of COVID-19 Patients at a Medical College Hospital in Bangladesh". Euroasian Journal of Hepato-Gastroenterology 10 (2020): 27-30.

23. Huq AKMF., et al. "Real-life Management Strategy of COVID-19 Patients in Bangladesh with No Death: An Observational and Cohort Study". Euroasian Journal of Hepato-Gastroenterology 10 (2020): 31-35.

24. Coronavirus is a failure of global governance - now the world needs a radical transformation" (2020).

25. Cai Q., et al. "Experimental Treatment with Favipiravir for COVID-19: An Open-Label Control Study”. Engineering (2020).

\section{Assets from publication with us}

- Prompt Acknowledgement after receiving the article

- Thorough Double blinded peer review

- Rapid Publication

- Issue of Publication Certificate

- High visibility of your Published work

Website: www.actascientific.com/

Submit Article: www.actascientific.com/submission.php

Email us: editor@actascientific.com

Contact us: +919182824667 